\title{
Pensamiento Aleatorio y Resolución de problemas en la Educación Básica Secundaria Normativa Curricular. Una Mirada Histórica ${ }^{1}$
}

\author{
Nubia Yaneth Gómez Velasco ${ }^{2}$ \\ Universidad Pedagógica y Tecnológica de Colombia-UPTC \\ Diana Carolina Beltrán Castañeda ${ }^{3}$ \\ Universidad Pedagógica y Tecnológica de Colombia-UPTC
}

Recepción: 14/08/2020

Evaluación: 17/08/2020

Aceptación: 15/10/2020

Artículo de Investigación-Científica

DOI: https://doi.org/10.22267/rhec.202525.85

\section{Resumen}

Bajo la premisa declarada por la Unesco, en la que constituye a la educación como la base firme sobre la cual se ha de edificar el progreso económico y social de un país (Martínez Boom, Noguera R. y Castro, 1988), en Colombia la Educación se concibe como un proceso constante de formación personal, cultural, social e integral del ser humano con sus derechos y deberes (Ministerio de Educación Nacional, 2020). En el ámbito educativo institucional, el currículo se estima como el conjunto de conocimientos que un estudiante debe adquirir para conseguir un determinado título académico (Oxford Léxico, 2020) y, en este contexto, Colombia ha experimentado la transformación de criterios en descripción y aplicación curricular para las instituciones educativas, en torno a situaciones sociales,

1 Este artículo se deriva de la investigación titulada: "Estrategias usadas por estudiantes de grado noveno para resolver problemas del Pensamiento Aleatorio en preguntas de selección múltiple con única respuesta”, avalada por la Universidad Pedagógica y Tecnológica de Colombia, UPTC, Colombia.

2 Docente de la Universidad Pedagógica y Tecnológica de Colombia-UPTC, Colombia. Doctora en Ciencias de la Educación. Grupo de investigación: GAMMA. Líneas de investigación: Métodos Estadísticos y Políticas Educativas. Correo electrónico: nubia. gomez@uptc.edu.co. (1) https://orcid.org/0000-0001-7745-172

3 Estudiante de Maestría Educación Matemática, Universidad Pedagógica y Tecnológica de Colombia-UPTC, Colombia. Grupo de investigación: GAMMA. Líneas de investigación: Didáctica. Correo electrónico: diana.beltran04@uptc.edu.co. (1) https:// orcid.org/ 0000-0002-3508-2451 
políticas, económicas y demográficas del país. Este artículo, ilustra una mirada histórica en materia curricular colombiana, con especial atención al desarrollo normativo del Pensamiento Aleatorio y la Resolución de Problemas en la Educación Básica Secundaria. Mediante una investigación cualitativa de tipo documental exploratorio, se concibe a la Historia como parte fundamental en el proceso de formación de la educación en Colombia.

Palabras clave: currículo; educación; normativa; Pensamiento Aleatorio; resolución.

\title{
Aleatory Thinking and Problem-solving strategies in Elementary and Secondary Schools. A historical perspective
}

\begin{abstract}
Under the premise declared by Unesco, which constitutes education as the strongest foundation on which the economic and social progress of a country is to be built (Martínez Boom, Noguera R. and Castro, 1988), education in Colombia is conceived as a constant process of personal, cultural, social and integral formation of human beings with their rights and duties (Ministry of National Education, 2020). In the institutional educational environment, the curriculum is considered as the set of knowledge that a student must acquire to achieve a certain academic degree (Oxford Lexicon, 2020) and, in this context, Colombia has experienced the transformation of criteria in the description and curricular application for educational institutions, around social, political, economic and demographic situations of the country. This article illustrates a historical perspective at Colombian curricular issues, with special attention to the normative development of Aleatory Thinking and Problem Solving in Elementary and Secondary Education. Through a qualitative research of exploratory documentary type, History is conceived as a fundamental part of the educational formation process in Colombia.
\end{abstract}

Keywords: curriculum; education; education regulations; aleatory thinking; educational resolution. 


\section{Pensamento Aleatório e Resolução de Problemas nos Regulamentos Curriculares do Ensino Secundário Básico. Um olhar histórico}

\section{Resumo}

Sob a premissa declarada pela Unesco, que constitui a educação como a base firme sobre a qual o progresso econômico e social de um país deve se construir (Martínez Boom, Noguera R. e Castro, 1988), na Colômbia a Educação é concebida como um processo constante da formação pessoal, cultural, social e integral do ser humano com seus direitos e deveres (Ministério da Educação Nacional, 2020). No campo educacional institucional, o currículo é considerado como o conjunto de conhecimentos que um aluno deve adquirir para atingir um determinado título acadêmico (Oxford Léxico, 2020) e, neste contexto, a Colômbia passou por uma transformação de critérios na descrição e aplicação curricular. para instituições de ensino, em torno das situações sociais, políticas, econômicas e demográficas do país. Este artigo ilustra um olhar histórico sobre os assuntos curriculares colombianos, com atenção especial para o desenvolvimento normativo do pensamento aleatório e solução de problemas no ensino médio. Por meio de pesquisas qualitativas de tipo documental exploratório, a História é concebida como parte fundamental do processo de formação educacional na Colômbia.

Palavras-chave: currículo; educação; normativa; pensamento aleatório; resolução.

\section{Introducción}

Desde el relato histórico que presenta esta descripción normativa, se convocan criterios de una reflexión nacional acerca de la educación como valor vital del ser y transformador de una sociedad, ya que, al constituirse por sus bases y proyecciones de enseñanza, nos precisan que, en Colombia, aún hay mucho por recorrer (Baena, 2011). 
Actualmente, el panorama internacional, evidenciado por resultados de pruebas externas que evalúan capacidades para aplicar conocimientos de estudiantes de Básica Secundaria, permite ver que Colombia enfrenta grandes retos en educación matemática (Schleicher, 2019; Ayala, 2015; Icfes, 2017a; 2020a; Ortiz et al., 2017). Por esta razón, se han fortalecido los instrumentos de evaluación en calidad educativa (MEN, 2009), a través del Instituto Colombiano para la Evaluación de la Educación - Icfes (Rama Ejecutiva Educación Nacional, 2010; Icfes, 2017b), que ponen en marcha los criterios construidos durante el trayecto histórico descrito en esta revisión documental (Icfes, 2017c; 2019; 2020b). Algunos ejemplos de su aplicación se presentan en Icfes (2016a; 2016b).

En Colombia, el carácter no determinístico de la estadística en la resolución de problemas comprende la certidumbre de diferentes opciones de respuesta y precisa que el aprendizaje determinado por la enseñanza y la evaluación de los estudiantes les permite identificar conceptos de aleatoriedad y certidumbre para argumentar, interpretar y tomar decisiones (MEN, 1998). Esta enseñanza se complementa a partir del conocimiento de errores y dificultades en la comprensión de conceptos estadísticos (Batanero, Godino y Green, 2014).

A través del currículo, se definen las habilidades a desarrollar para que el estudiante conozca lo que puede hacer en situaciones de contexto diferentes a las identificadas en el aula (MEN, 2006).

Estas habilidades se traducen en competencias, definidas como las metas que indican lo que el estudiante debe saber y puede hacer con lo que debe saber; en otras palabras, lo que el estudiante debe aprender y saber aplicar en diversos contextos. Su origen surge con los indicadores de logros curriculares, que equivalen a las metas definidas por el Ministerio de Educación Nacional, que deben cumplir los estudiantes (1996). Luego, estos lineamientos curriculares (1998) introducen el término de estándar con el objeto de mejorar el desempeño de los estudiantes, definir expectativas de aprendizaje, unificar criterios de enseñanza en las instituciones educativas, direccionar esfuerzos hacia una calidad mayor, identificar niveles de desempeño y competencias a evaluar por grado escolar, así como facilitar el proceso de evaluación (MEN, 2002b).

Asimismo, los estándares de competencias se diseñaron para que, con claridad, el sistema educativo identifique a dónde quiere ir como punto de llegada y cuán lejos se encuentra de la meta deseada (para diseñar 
estrategias de mejoramiento), y para definir el punto de partida y marco referencial en el diseño de planes, programas y actividades institucionales (MEN, 2002a). Con este propósito, surgen la renovación, revolución y actualización curricular de 2002, 2003 y 2006, respectivamente. Estos aspectos se encuentran vinculados a políticas educativas, como las políticas de ciencia y tecnología a nivel de Colombia (Gómez, Soto y Lima, 2018).

Esta investigación, de tipo documental, exploratoria, presenta, en orden, los hechos desde el siglo XX hasta inicios del siglo XXI, en materia normativa de la Historia curricular en Colombia.

La revisión documental se apoyó en la información virtual del MEN y otros autores, tal como se ilustra en la lista de referentes bibliográficos dispuestos al final de este documento, que constituyen el cuerpo de resultados. Esta revisión documental visualiza un crecimiento importante de publicaciones científicas sobre estos temas, crecimiento de producción científica de tipo exponencial, como se observa en otros estudios (Gómez y Rodríguez, 2014; Gómez et al., 2014, Gómez et al., 2020).

El objetivo de este artículo es presentar un análisis sobre la revisión documental de los cambios curriculares que se han presentado en Colombia, a partir de una descripción general, desde 1930, hasta las características normativas vigentes, complementado con la descripción de directrices del currículo en torno al Pensamiento Aleatorio y Resolución de Problemas en Básica Secundaria.

Este análisis podrá servir de referencia a la comunidad académica para comprender los fundamentos teóricos de la evolución curricular en Colombia, en condiciones sociales, políticas y económicas asociadas con sus modificaciones; en particular, lo relacionado con la Resolución de Problemas y el Pensamiento Aleatorio en la Educación Básica Secundaria.

Los resultados de este artículo ilustran una síntesis de la evolución histórica curricular colombiana y, luego, con base en los sucesos de 1996, 1998, 2002, 2003 y 2006, se describe la normativa respecto al Pensamiento Aleatorio y la Resolución de Problemas en Básica Secundaria, para finalizar con algunas conclusiones.

Este artículo forma parte del proyecto de investigación Estrategias usadas por estudiantes de grado noveno para resolver problemas del Pensamiento Aleatorio en preguntas de selección múltiple. La propuesta se viene desarrollando en el grupo de investigación en Estadística Gamma, a través del programa de Maestría en Educación Matemática 
de la Universidad Pedagógica y Tecnológica de Colombia, vinculado al programa Beca Maestra, del Departamento de Boyacá, en Colombia, por iniciativa del MEN, Colciencias y la Gobernación de Boyacá.

\section{Metodología}

Esta investigación, de tipo documental exploratorio, ha seleccionado y acopiado información a través de la lectura crítica de documentos y materiales bibliográficos que permiten construir conocimiento en un proceso más amplio de investigación y de ordenamiento de sus referentes teóricos (Baena, 1985; Vivero y Sánchez, 2018). Es una investigación de tipo documental, que permite profundizar en la temática objeto de estudio, a partir de información encontrada en entidades oficiales y gubernamentales, como fuente de análisis.

Desde la perspectiva metodológica abordada, este ejercicio científico contribuye a disponer información para establecer nuevos planteamientos de aprendizaje a partir de su recorrido histórico, que posibilite esclarecer comportamientos y tendencias dadas sobre un suceso, que tiene implicaciones en el presente (Gómez, et al., 2020b).

En esta investigación, se realizó un análisis, síntesis y selección de información, mediante la búsqueda en internet y el acceso a documentos científicos y repositorios digitales, con los cuales se presenta la construcción de nuevos conocimientos, de forma ordenada y selectiva (QuestionPro, 2020). La principal fuente de información es el MEN, entre otras, con lo que se construyeron las tablas presentadas en la sección de resultados.

\section{Resultados}

En principio, se describe un panorama histórico de normativas curriculares en Colombia; luego, se enfatiza en las normativas Curriculares Colombiana en Básica Secundaria, con el interés en el objeto de estudio, referente a Pensamiento Aleatorio y Resolución de problemas.

\subsection{Panorama histórico de normativas curriculares en Colombia}

Al Sistema educativo nacional vigente en Colombia lo forman: educación inicial, educación preescolar, educación básica (primaria, grados, $1^{\circ}$ a $5^{\circ}, \mathrm{y}$ 
secundaria, grados $6^{\circ}$ a $9^{\circ}$ ), educación media (grados $10^{\circ}$ y $11^{\circ}$, para obtener el título de bachiller) y la Educación Superior (MEN, 2020). El MEN, como ente regulador y administrativo del Sistema educativo colombiano, en ejercicio de sus facultades, ha formulado criterios y normas para organizarlo en función de la construcción social que representa la educación.

El panorama histórico del trayecto curricular en Colombia presenta aspectos relevantes, que datan de 1930 (Pineda et al., 2017), año que se tomará de referencia, en esta revisión histórica, para la revisión de las propuestas curriculares en Colombia, con una breve descripción de sus principales características y la elaboración de un esquema que detalla los principales cambios de la época.

En el Cuadro 1, se describe en orden cronológico la normativa establecida por el MEN, en Colombia, y la descripción de aportes a la renovación curricular.

Cuadro 1. Normativas curriculares colombianas. Mirada histórica.

\begin{tabular}{|c|l|}
\hline Evento o referente normativo & \multicolumn{1}{|c|}{ Descripción del aporte asociado al currículo } \\
\hline De 1930 a 1950 & $\begin{array}{l}\text { La propuesta curricular considera formar un buen } \\
\text { ciudadano, en educación primaria, y capacitarlo en } \\
\text { conceptos culturales, intelectuales, morales y éticos, en } \\
\text { educación secundaria. }\end{array}$ \\
\hline De 1950 a 1970 & $\begin{array}{l}\text { Se denomina la época de la expansión educativa, con la } \\
\text { mejora de los indicadores de la educación, con base en } \\
\text { factores políticos, demográficos y económicos. Se observa } \\
\text { la no consecución de evaluaciones memorísticas y una } \\
\text { equilibrada formación de los aspectos humano y técnico. }\end{array}$ \\
\hline $\begin{array}{c}\text { De 1957 a 1962 } \\
\text { Edimer Quinquenal de }\end{array}$ & $\begin{array}{l}\text { Se propuso la duración de cinco años en primaria, para el } \\
\text { sector rural, así como el desarrollo de líneas de formación } \\
\text { técnica, universitaria y Normal, en la educación secundaria. }\end{array}$ \\
\hline $\begin{array}{c}\text { Resolución } \mathbf{8 0 7} \text { de 1961 } \\
\text { Inspector escolar departamental }\end{array}$ & $\begin{array}{l}\text { Brinda asesoría a docentes para uso adecuado de objetivos } \\
\text { y programas educativos. }\end{array}$ \\
\hline $\begin{array}{c}\text { Decreto 1710 de 1963 } \\
\text { Educación Primaria colombiana y y } \\
\text { dicta otras disposiciones }\end{array}$ & $\begin{array}{l}\text { Unifica la escuela primaria que, desde 1950, establecía } \\
\text { un triple Sistema educativo, en primaria. En matemáticas, } \\
\text { enfatiza en aritmética y geometría intuitiva (MEN, 1963). }\end{array}$ \\
\hline
\end{tabular}




\begin{tabular}{|c|c|}
\hline $\begin{array}{c}\text { De } 1965 \text { a } 1975 \\
\text { Misión Pedagógica Alemana }\end{array}$ & $\begin{array}{l}\text { Se establecen medidas que normalizan el currículo } \\
\text { nacional en nivel y calidad de estudios, para mejorar el } \\
\text { Sistema educativo mediante guías didácticas alemanas, } \\
\text { que contienen: tema, subtemas, objetivos, introducción, } \\
\text { contenido, estrategias pedagógicas, material, actividades, } \\
\text { evaluaciones, tareas, consultas, técnicas. Esta propuesta } \\
\text { la lideró la Misión Pedagógica Alemana, compuesta por } \\
25 \text { colombianos, técnicos educativos en primaria, y } 6 \\
\text { alemanes, de nivel educativo superior. }\end{array}$ \\
\hline De 1970 a 1974 & $\begin{array}{l}\text { Incursiona el currículo basado en el instruccionismo de la } \\
\text { tecnología educativa estadounidense, con implementación } \\
\text { de la modalidad vocacional en pedagógica, industrial, } \\
\text { agropecuaria, comercial, salud y nutrición, educación } \\
\text { física y recreación, y promoción de la comunidad. Esto } \\
\text { se dispuso para favorecer el progreso del país en temas } \\
\text { laborales y económicos. }\end{array}$ \\
\hline $\begin{array}{c}\text { Decreto } 088 \text { de } 1976 \\
\text { Reestructura el Sistema educativo } \\
\text { y reorganiza el MEN }\end{array}$ & $\begin{array}{l}\text { Concede redefinir objetivos en planes y programas } \\
\text { curriculares. Sobre la base de modelos foráneos, de origen } \\
\text { tecnocrático y conductista, la educación se centró en el } \\
\text { currículo, la enseñanza e instrucción y la evaluación: } \\
\text { Taylorización y didactismo en la educación (MEN, 1976). }\end{array}$ \\
\hline $\begin{array}{c}\text { Decreto } 1419 \text { de } 1978 \\
\text { Reestructura el Sistema educativo y } \\
\text { reorganiza el MEN }\end{array}$ & $\begin{array}{l}\text { Define el concepto, características generales y por niveles, } \\
\text { fines institucionales y componentes del currículo. Esta } \\
\text { reforma curricular modificó la estructura del Sistema } \\
\text { educativo, planes de enseñanza, práctica pedagógica, } \\
\text { organización del sector educativo y prestación de servicios } \\
\text { del Estado (MEN, 1978). }\end{array}$ \\
\hline $\begin{array}{c}\text { Inicios de los años } 80 \\
\text { Plan de Integración Nacional }\end{array}$ & $\begin{array}{l}\text { La perspectiva curricular colombiana apoya la educación } \\
\text { formal, no formal e informal. En nivel de básica secundaria, } \\
\text { incursiona en cobertura para desarrollar habilidades } \\
\text { laborales, acordes a la vocación productiva del sector, } \\
\text { además de las culturales, científicas y tecnológicas. }\end{array}$ \\
\hline $\begin{array}{c}\text { Decreto } 1002 \text { de } \mathbf{1 9 8 4} \\
\text { Establece el Plan de Estudios para } \\
\text { la Educación Preescolar, Básica } \\
\text { (primaria y secundaria) y Media } \\
\text { Vocacional de la Educación Formal } \\
\text { colombiana }\end{array}$ & $\begin{array}{l}\text { Describe los objetivos de aprendizaje por nivel educativo } \\
\text { con aplicación generalizada, a partir de lo cual se considera } \\
\text { el objetivo que indica al estudiante: la participación crítica } \\
\text { y creativa en la solución de problemas y en el desarrollo de } \\
\text { su comunidad (MEN, 1984a). }\end{array}$ \\
\hline $\begin{array}{c}\text { Decreto } 2647 \text { de } \mathbf{1 9 8 4} \\
\text { Fomenta las innovaciones } \\
\text { educativas en el Sistema Educativo } \\
\text { Nacional } \\
\text { Renovación curricular }\end{array}$ & $\begin{array}{l}\text { Nuevo currículo, planteado desde necesidades del } \\
\text { estudiante, sobre cuatro ejes: académico, pedagógico, } \\
\text { administrativo y financiero. Con estrategias de desarrollo } \\
\text { en investigación científica y tecnológica; desarrollo } \\
\text { cultural, recreacional y deportivo; y eficiencia en uso de } \\
\text { recursos financieros. Asimismo, fomentó la creatividad } \\
\text { para resolver problemas (MEN, 1984b). }\end{array}$ \\
\hline
\end{tabular}




\begin{tabular}{|c|c|}
\hline $\begin{array}{c}\text { Ley } 24 \text { de } 1988 \\
\text { Reestructura el MEN y dicta otras } \\
\text { disposiciones } \\
\text { Renovación curricular }\end{array}$ & $\begin{array}{l}\text { Fortalece la política educativa sobre renovación curricular, } \\
\text { con vinculación de componentes estratégicos, según } \\
\text { características y necesidades en contextos regional y } \\
\text { cultural (MEN, 1988). }\end{array}$ \\
\hline $\begin{array}{c}\text { Movimiento Pedagógico } \\
\text { Inicios de años } 90 \\
\text { Mentes colombianas investigadoras } \\
\text { con fundamentos en concepciones } \\
\text { epistemológicas y pedagógicas de } \\
\text { Habermas, Wittgenstein, Kuhn, } \\
\text { Bernstein }\end{array}$ & $\begin{array}{l}\text { Con el Centro de Estudios e Investigaciones Docentes y } \\
\text { la revista Educación y Cultura, como medios de expresión } \\
\text { de la intelectualidad colombiana, motiva reformas } \\
\text { para fortalecer autonomía en instituciones educativas y } \\
\text { liderazgo docente en procesos educativo, pedagógico, } \\
\text { didáctico y curricular. }\end{array}$ \\
\hline $\begin{array}{r}\text { Ley } 115 \\
\text { Ley General d }\end{array}$ & $\begin{array}{l}\text { Confiere autonomía a las instituciones educativas para } \\
\text { definir el currículo, que establece como el conjunto de } \\
\text { criterios, planes de estudio, programas y procesos, para } \\
\text { alcanzar objetivos del Proyecto Educativo Institucional } \\
\text { (PEI, Art. 76). } \\
\text { Establece orientaciones para el proceso de construcción del } \\
\text { currículo en las instituciones educativas y los indicadores } \\
\text { de logros curriculares para la educación formal (Art. 78, } \\
\text { 148b). (MEN, 1994b). }\end{array}$ \\
\hline $\begin{array}{r}\text { Decreto 18 } \\
\text { Reglamenta aspec } \\
\text { y organizativos g } \\
115 / 1\end{array}$ & $\begin{array}{l}\text { Define las normas reglamentarias de prestación del servicio } \\
\text { educativo, organización de la educación formal, PEI, } \\
\text { gobierno escolar y organización institucional, orientaciones } \\
\text { curriculares, evaluación y promoción. (MEN, 1994a) }\end{array}$ \\
\hline $\begin{array}{c}\text { Resolución } 2343 \text { de } 1996 \\
\text { Adopta un diseño de lineamientos } \\
\text { generales de los procesos } \\
\text { curriculares del servicio público } \\
\text { educativo y establece indicadores } \\
\text { de logros curriculares para la } \\
\text { educación formal }\end{array}$ & $\begin{array}{l}\text { Dispone indicadores de logros curriculares para interpretar } \\
\text { (estimar, valorar, autorregular, controlar) los resultados } \\
\text { del proceso educativo (aspectos del desarrollo humano: } \\
\text { valores, actitudes, competencias, conocimientos), como } \\
\text { punto de partida para construir el currículo en la institución } \\
\text { educativa, según el PEI, lo cual significó, para los docentes, } \\
\text { cambiar la forma de planeación por logros e indicadores de } \\
\text { logro (currículos atomizados - por docente) (MEN, 1996). }\end{array}$ \\
\hline $\begin{array}{l}1998 \\
\text { Serie Lineamientos Curriculares } \\
\text { Revolución Curricular }\end{array}$ & El MEN publica Lineamientos para construir los currículos. \\
\hline $\begin{array}{c}\mathbf{2 0 0 2} \\
\text { Estándares curriculares } \\
\text { Lo que los estudiantes deben saber } \\
\text { y ser capaces de hacer en un área } \\
\text { y grado } \\
\end{array}$ & $\begin{array}{l}\text { El MEN define las normas básicas sobre competencias } \\
\text { y directrices pedagógicas - fase Renovación curricular } \\
\text { (MEN, 2002b). }\end{array}$ \\
\hline $\begin{array}{c}\mathbf{2 0 0 3} \\
\text { Estándares Básicos } \\
\text { Lo que un estudiante puede estar en } \\
\text { capacidad de saber y saber hacer }\end{array}$ & $\begin{array}{l}\text { El MEN establece normas básicas sobre competencias } \\
\text { y directrices pedagógicas - fase Revolución educativa } \\
(\mathrm{MEN}, 2003) \text {. }\end{array}$ \\
\hline
\end{tabular}




\begin{tabular}{|c|l|}
\hline $\begin{array}{c}\text { E006 } \\
\begin{array}{c}\text { Lo que los estudiantes deben saber } \\
\text { y saber hacer con lo que aprenden }\end{array}\end{array}$ & $\begin{array}{l}\text { El MEN consolida normas básicas sobre competencias y } \\
\text { directrices pedagógicas - fase Actualización curricular } \\
\text { (MEN, 2006). }\end{array}$ \\
\hline $\mathbf{2 0 1 5}$ & $\begin{array}{l}\text { Por medio del Programa de Transformación Educativa } \\
\text { "Programa Todos a Aprender" (PTA), brinda a las } \\
\text { instituciones educativas orientaciones más prescriptivas } \\
\text { en relación con currículos y pedagogía. Los DBA, con } \\
\text { fundamento en Lineamientos curriculares y Estándares } \\
\text { básicos de competencias, sirven para informar a docentes } \\
\text { y padres de familia sobre las competencias de aprendizaje } \\
\text { que deben adquirir los estudiantes (MEN, 2016a). }\end{array}$ \\
$\begin{array}{c}\text { Competencias de aprendizaje en } \\
\text { las áreas de matemáticas y lenguaje } \\
\text { por cada grado }\end{array}$
\end{tabular}

Fuente: esta investigación. Pineda y Loaiza, 2017. OECD y MEN, 2016.

\subsection{Normativa curricular colombiana en Básica Secundaria: Pensamiento Aleatorio y Resolución de Problemas}

El Pensamiento Aleatorio y la Resolución de Problemas son tópicos que se han venido incorporando y posicionando en el Sistema educativo colombiano, con sus normativas curriculares.

Algunos autores sostienen sobre la necesidad de una adecuada comprensión de la probabilidad y la estadística para resolver situaciones aleatorias, como las votaciones, las inversiones, entre otras (Begué et al., 2020), sobre la base de la relación que existe entre el desarrollo de un país y su Sistema estadístico, con información suficiente para tomar decisiones en aspectos económicos, sociales y políticos (Batanero, 2000), elementos que se visualizan en investigaciones de tipo colaborativo y potencializan el tratamiento de problemáticas en forma interdisciplinaria (Jiménez et al., 2018).

En las aulas, cada vez más, se precisa en la formación de ciudadanos competentes, con disposición, a través del Plan de estudios, del desarrollo de competencias en el pensamiento aleatorio, donde el estudiante interprete, analice y utilice resultados publicados en periódicos, revistas, televisión $\mathrm{u}$ hojas impresas, en los que indique situaciones posibles de un evento, estime si son o no probables, les asigne probabilidad numérica y considere aquellos elementos que le permitan acopiar, estudiar, resumir y representar sistemas de datos estadisticos para tomar decisiones ante la incertidumbre de un suceso (MEN, 2006), que son fundamentales en el pensamiento crítico (Gómez et al., 2019) 
En relación con esto, estos tópicos de conocimiento, además de ser fácilmente aplicables en la cotidianidad, por sus técnicas matemáticas no complicadas, ofrecen a los estudiantes la oportunidad de entender y resolver problemas de su entorno, apoyados en la enseñanza de la heurística para resolver problemas, cada día más importante para comprender las técnicas de análisis de datos y su óptima interpretación en la actual y futura sociedad (Batanero, 2001; Beltrán y Gómez, 2020).

El enfoque de Educación Matemática Realista (EMR) indica que resolver problemas en contextos cotidianos da sentido al aprendizaje en matemáticas (Departamento de Educación de la Universidad Central Chile, 2013), en Pensamiento Aleatorio, que se despierta en diferentes niveles de formación a través de diferentes actividades, como, entre otras, las desarrolladas por semilleros de investigación (Gómez y Jiménez, 2015).

En relación con esto, las normativas en Colombia muestran pautas sobre la importancia de estos tópicos de conocimiento. Como aparte destacable del currículo, en el nivel de educación básica secundaria alusivo al tema de investigación, se precisa que, en 1990, se incluyen las temáticas del conocimiento estadístico en ese nivel de formación (Castellanos Sánchez, Batanero y Arteaga Cezón, 2014). Sus referentes teóricos iniciales, por cada grado escolar de la Educación Básica Secundaria, fueron:

Grado $6^{\circ}$ : frecuencias absolutas y relativas, diagramas de barras y circular, frecuencias ordinarias y acumuladas;

Grado $7^{\circ}$ : medidas de tendencia central - media, mediana y moda; Grado $8^{\circ}$ : medición, muestreo, escala, disposición y representación de datos;

Grado 9: medidas de dispersión.

Con este referente y con base en la evolución histórica de la normativa curricular colombiana, ya presentada en la sección anterior, se ilustra, mediante representación en un cuadro, la descripción específica de los contenidos definidos en educación Básica Secundaria, para cada tópico del Pensamiento Aleatorio y la Resolución de Problemas: indicadores de logros curriculares (1996), Lineamientos curriculares (1998), Estándares curriculares (2002), Estándares básicos (2003) y Estándares básicos de competencias (2006). 


\subsubsection{Indicadores de Logros Curriculares en Básica Secundaria. Pensamiento Aleatorio y Resolución de Problemas}

En alusión a lo descrito en la sección de la normativa histórica, el Cuadro 2 presenta los indicadores de logros curriculares asociados al Pensamiento Aleatorio y la Resolución de Problemas, en la Educación Básica Secundaria, que comprende los grados $6^{\circ}, 7^{\circ}, 8^{\circ}, 9^{\circ}$.

Cuadro 2. Indicadores de logros Curriculares en Colombia, para Educación Básica Secundaria. Pensamiento Aleatorio y Resolución de Problemas.

\begin{tabular}{|c|c|c|}
\hline Grado & $\begin{array}{c}\text { Pensamiento aleatorio y } \\
\text { sistemas de datos }\end{array}$ & Resolución de problemas \\
\hline 6 & $\begin{array}{l}\text { - Interpreta datos presentados en tablas y } \\
\text { en diagramas, comprende y usa la media, la } \\
\text { mediana y la moda en un conjunto pequeño } \\
\text { de datos y saca conclusiones estadísticas. } \\
\text { - Reconoce la importancia de averiguar datos } \\
\text { y procesar información para tomar decisiones, } \\
\text { y de conocer y evaluar sus características en } \\
\text { relación con las decisiones que se tomen. }\end{array}$ & $\begin{array}{l}\text { - Investiga y comprende contenidos } \\
\text { matemáticos a partir de enfoques de } \\
\text { resoluciones de problemas, formula } \\
\text { y resuelve problemas derivados de } \\
\text { situaciones cotidianas y matemáticas, } \\
\text { examina los resultados teniendo en } \\
\text { cuenta el planteamiento original del } \\
\text { problema. }\end{array}$ \\
\hline $7^{\circ}, 8^{\circ}, 9^{\circ}$ & $\begin{array}{l}\text { - Relaciona orden de parejas, ternas ordenadas, } \\
\text { cuaternas ordenadas, combinaciones de } \\
\text { elementos entre conjuntos. } \\
\text { - Analiza cualitativamente gráficas con } \\
\text { rectas y curvas continuas y escalonadas, sus } \\
\text { características y efectos en las gráficas y el } \\
\text { cambio de parámetros. }\end{array}$ & $\begin{array}{l}\text { - Usa enfoque de solución de } \\
\text { problemas, investigando contenidos } \\
\text { matemáticos y desarrollando } \\
\text { estrategias para resolverlos. } \\
\text { - Formula hipótesis, las modifica, las } \\
\text { descarta y las argumenta analizando } \\
\text { y resolviendo problemas. }\end{array}$ \\
\hline
\end{tabular}

Fuente: esta investigación; MEN, 1996, 37-38, 42-43.

\subsubsection{Lineamientos Curriculares en Básica Secundaria. Pensamiento Aleatorio y Resolución de Problemas}

Los lineamientos Curriculares indican que el Pensamiento Aleatorio es aplicable a la certidumbre aproximada de sucesos inciertos (juegos del azar); sugiere incluir la investigación en el proceso de aprendizaje en el aula; integrar la modelación de fenómenos físicos con estrategias de simulación en experimentos y conteo; y evaluar diversas aproximaciones en contextos cotidianos (MEN, 1998). El Cuadro 3 ilustra orientaciones a procesos estadísticos. 
Cuadro 3. Lineamientos Curriculares para el Pensamiento Aleatorio.

\begin{tabular}{|c|c|}
\hline $\begin{array}{r}\text { Proceso d } \\
\text { análisis }\end{array}$ & Proceso de búsqueda y acopio de datos \\
\hline $\begin{array}{ll}\checkmark & \text { Saber cuánta } \\
\text { información se requiere. } \\
\checkmark & \text { Saber acopiarla } \\
& \text { (metodología). } \\
\checkmark & \text { Saber representarla } \\
& \text { (información recogida). } \\
\checkmark & \text { Saber interpretarla } \\
& \text { (información recogida). }\end{array}$ & $\begin{array}{l}\square \text { Definir objetivos acerca del por qué y para qué acopiar dicha } \\
\text { información, así como el dónde y cuándo. } \\
\square \text { Elaborar lista de posibles respuestas a encontrar. } \\
\square \text { Identificar posibles dificultades durante el proceso de acopio. } \\
\text { Variables en el entorno que pudieran impedir la consecución de } \\
\text { logros u objetivos en la búsqueda de información. } \\
\square \text { Organizar fuentes de búsqueda y hallazgo de información objeto. } \\
\square \text { Evaluar (analizar, comprobar) la veracidad de la información } \\
\text { identificada, hallada o resultante de búsqueda. } \\
\square \text { Evaluar la ética en el acopio (veracidad). }\end{array}$ \\
\hline
\end{tabular}

Fuente: esta investigación; MEN, 1998, 47-48.

Respecto a la resolución de problemas, aunado con la perspectiva de Chile y España, según Benavides et al., (2004), en Colombia, los Lineamientos precisan la importancia de elegir los contextos que correspondan a las experiencias y vivencias del entorno del estudiante, para disponer de sus habilidades y estrategias en la resolución. El Cuadro 4 presenta estas sugerencias:

Cuadro 4. Lineamientos Curriculares para incluir la Resolución de Problemas.

\section{Lineamientos curriculares de la resolución en problemas matemáticos}

Promover la formulación de problemas en contextos matemáticos (del área) y no matemáticos.

Presentar y aplicar estrategias para resolver problemas.

Comparar la solución (teórica en el aula) con la solución en contextos reales.

Definir criterios generales en solución de problemas para situaciones futuras.

Fuente: esta investigación; MEN, 1998, 52.

\subsubsection{Estándares Curriculares en Básica Secundaria. Pensamiento Aleatorio y Resolución de Problemas}

La renovación curricular del 2002 (MEN, 2002b) establece estándares curriculares definidos como "lo que los estudiantes deben saber y ser capaces de hacer por área y grado escolar”. Estos estándares se describen en el Cuadro 5, respecto a lo establecido por grado para desarrollar el Pensamiento Aleatorio y fortalecer habilidades en resolución de problemas, 


\section{en formación básica secundaria (grados $6^{\circ}$ a $9^{\circ}$ ). Estos estándares tratan temas básicos en la formación de un estudiante y contribuyen a formar una cultura estadística, que le permitan interpretar situaciones de contexto, como las que se reportan en el día a día en diferentes casos.}

Cuadro 5. Estándares Curriculares en la Educación Básica Secundaria. Pensamiento Aleatorio y Resolución de Problemas

\begin{tabular}{|c|c|c|}
\hline Grado & $\begin{array}{c}\text { Pensamiento aleatorio y sistemas } \\
\text { de datos }\end{array}$ & Resolución de problemas \\
\hline $6^{\circ}$ & $\begin{array}{l}\text { - Construye diagramas de barras, } \\
\text { diagramas circulares y pictogramas a } \\
\text { partir de una colección de datos. } \\
\text { - Interpreta diagramas de barras, } \\
\text { diagramas circulares y pictogramas y } \\
\text { calcula frecuencias, medianas, modas } \\
\text { y medias a partir de ellas. }\end{array}$ & $\begin{array}{l}\text { - Resuelve problemas no rutinarios, } \\
\text { mediante la selección de conceptos y } \\
\text { técnicas matemáticas apropiadas. }\end{array}$ \\
\hline $7^{\circ}$ & $\begin{array}{l}\text { - Identifica el término "probabilidad" } \\
\text { como un número entre cero y uno, } \\
\text { que indica qué tan probable es que un } \\
\text { evento ocurra. } \\
\text { - Calcula la probabilidad de algunos } \\
\text { eventos sencillos. } \\
\text { - Hace inferencias significativas a } \\
\text { partir de la moda, la mediana y la } \\
\text { media de una colección de datos. }\end{array}$ & $\begin{array}{l}\text { - Formula problemas matemáticos } \\
\text { en el contexto de otras disciplinas y } \\
\text { los resuelve con los conocimientos y } \\
\text { herramientas adquiridas. }\end{array}$ \\
\hline $8^{\circ}$ & $\begin{array}{l}\text { - Encuentra el mínimo, máximo, rango } \\
\text { y rango intercuartil de una colección } \\
\text { de datos y deduce inferencias } \\
\text { significativas de esta información. } \\
\text { - Identifica el espacio muestral de } \\
\text { un experimento sencillo y calcula } \\
\text { probabilidad de eventos sencillos. }\end{array}$ & $\begin{array}{l}\text { - Traduce problemas del lenguaje } \\
\text { común al algebraico y los resuelve } \\
\text { satisfactoriamente. }\end{array}$ \\
\hline $9^{\circ}$ & $\begin{array}{l}\text { - Interpreta diagramas, encuestas, } \\
\text { gráficas y tablas que recojan datos de } \\
\text { asuntos cotidianos y hace inferencias } \\
\text { y predicciones a partir de éstos. } \\
\text { - Comprende y aplica las medidas } \\
\text { de tendencia central en el análisis de } \\
\text { datos de diversa índole. }\end{array}$ & $\begin{array}{l}\text { - Resuelve problemas cada vez más } \\
\text { complejos, descomponiéndolos en } \\
\text { partes más sencillas y aplicando una } \\
\text { diversidad de estrategias. } \\
\text { - Hace generalizaciones de las } \\
\text { soluciones que obtiene. } \\
\text { - Utiliza de manera creativa } \\
\text { una calculadora científica o graficadora } \\
\text { para llevar a cabo experimentos, probar } \\
\text { conjeturas y resolver problemas. }\end{array}$ \\
\hline
\end{tabular}

Fuente: esta investigación; MEN, 2002b, 28-37. 


\subsubsection{Estándares Básicos en Básica Secundaria. Pensamiento Aleatorio y Resolución de Problemas}

Con la revolución matemática de 2003, los estándares se modifican y los definen bajo el título "Lo que un estudiante puede estar en capacidad de saber y saber hacer, en determinada área y en determinado nivel" (MEN, 2003). Con este referente, se busca enfatizar en estos estándares, un tratamiento de los conceptos en un contexto y que fuese motivador para el estudiante. En el Cuadro 6, se presentan los estándares para el Pensamiento Aleatorio y la Resolución de Problemas en la educación básica secundaria.

Cuadro 6. Estándares Básicos en la Educación Básica Secundaria. Pensamiento Aleatorio y Resolución de Problemas.

\begin{tabular}{|c|c|c|}
\hline Grado & $\begin{array}{c}\text { Pensamiento aleatorio y sistemas } \\
\text { de datos }\end{array}$ & Resolución de problemas \\
\hline $6^{\circ}-7^{\circ}$ & $\begin{array}{l}\text { 1. Comparar e interpretar datos } \\
\text { provenientes de diversas fuentes } \\
\text { (prensa, revistas, televisión, } \\
\text { experimentos, consultas, entrevistas). } \\
\text { 2. Reconocer la relación entre un } \\
\text { conjunto de datos y surepresentación. } \\
\text { 3. Usar representaciones gráficas } \\
\text { adecuadas para presentar diversos } \\
\text { tipos de datos (diagramas de barras, } \\
\text { diagramas circulares). } \\
\text { 4. Usar medidas de tendencia } \\
\text { central (media, mediana, moda) para } \\
\text { interpretar el comportamiento de un } \\
\text { conjunto de datos. } \\
\text { 5. Usar modelos (diagramas de } \\
\text { árbol, por ejemplo) para discutir y } \\
\text { predecir la posibilidad de ocurrencia } \\
\text { de un evento. } \\
6 . \text { Hacer conjeturas acerca del } \\
\text { resultado de un experimento aleatorio } \\
\text { usando proporcionalidad y nociones } \\
\text { básicas de probabilidad. }\end{array}$ & $\begin{array}{l}\text { 7. Resolver y formular problemas } \\
\text { a partir de un conjunto de datos } \\
\text { presentados en tablas, diagramas de } \\
\text { barras, diagramas circulares. }\end{array}$ \\
\hline & $\begin{array}{l}\text { 8. Predecir y justificar razonamientos } \\
\text { y conclusiones usando información } \\
\text { estadística. }\end{array}$ & \\
\hline
\end{tabular}




\begin{tabular}{|c|c|c|}
\hline $8^{\circ}-9^{\circ}$ & $\begin{array}{l}\text { 1. Reconocer que, diferentes maneras } \\
\text { de presentar la información pueden } \\
\text { dar origen a distintas interpretaciones. } \\
\text { 2. Interpretar analítica y críticamente } \\
\text { información estadística proveniente } \\
\text { de diversas fuentes (prensa, revistas, } \\
\text { televisión, experimentos, consultas, } \\
\text { entrevistas). } \\
\text { 3. Interpretar conceptos de media, } \\
\text { mediana yoda. } \\
\text { 4. Seleccionar y usar algunos } \\
\text { métodos estadísticos adecuados } \\
\text { según el tipo de información. } \\
\text { 5. Comparar resultados } \\
\text { experimentales con probabilidad } \\
\text { matemática esperada. } \\
\text { 7. Reconocer tendencias que se } \\
\text { presentan en conjuntos de variables } \\
\text { relacionadas. } \\
\text { 8. Calcular probabilidad de eventos } \\
\text { simples usando métodos diversos } \\
\text { (listados, diagramas de árbol, } \\
\text { técnicas de conteo). básicos de } \\
\text { 9. Usar conceptos basicios muestral, } \\
\text { probabilidad (espacio mad } \\
\text { evento, independencia...) }\end{array}$ & $\begin{array}{l}\text { 6. Resolver y formular problemas } \\
\text { seleccionando información } \\
\text { relevante en conjuntos de datos } \\
\text { provenientes de fuentes diversas } \\
\text { (prensa, revistas, televisión, } \\
\text { experimentos, consultas, } \\
\text { entrevistas). }\end{array}$ \\
\hline
\end{tabular}

Fuente: esta investigación; MEN, 2003, 22-27.

\subsubsection{Estándares Básicos de Competencias en Básica Secundaria. Pensamiento Aleatorio y Resolución de Problemas}

La actualización curricular define estándares por competencias como "lo que los estudiantes deben saber y saber hacer con lo que aprenden" (MEN, 2006). En educación Básica Secundaria (grado $6^{\circ}$ a $9^{\circ}$ ), se unifican por cada dos grados escolares. Al considerar el nivel de competencias previsto a alcanzar según el MEN, se unifican, por grupos, competencias a adquirir en relación con el pensamiento aleatorio y resolución de problemas, con reiteración del énfasis en la aplicación en contexto, con un análisis interpretativo y argumentativo de la situación ilustrada.

El Cuadro 7 presenta esta descripción para el Pensamiento Aleatorio y la Resolución de problemas. 


\section{Cuadro 7. Estándares Básicos de Competencias en la Educación Básica Secundaria. Pensamiento Aleatorio y Resolución de Problemas}

\begin{tabular}{|c|c|c|}
\hline Grado & Pensamiento aleatorio y sistemas de datos & $\begin{array}{c}\text { Resolución de } \\
\text { problemas }\end{array}$ \\
\hline $6^{\circ}-7^{\circ}$ & $\begin{array}{l}\text { - Comparo e interpreto datos provenientes de diversas } \\
\text { fuentes (prensa, revistas, televisión, experimentos, } \\
\text { consultas, entrevistas). } \\
\text { - Reconozco la relación entre un conjunto de datos y su } \\
\text { representación. } \\
\text { - Interpreto, produzco y comparo representaciones } \\
\text { gráficas adecuadas para presentar diversos tipos de datos } \\
\text { (diagramas de barras, diagramas circulares). } \\
\text { - Uso medidas de tendencia central (media, mediana, } \\
\text { moda) para interpretar el comportamiento de un conjunto } \\
\text { de datos. } \\
\text { - Uso modelos (diagramas de árbol, por ejemplo) para } \\
\text { discutir y predecir la posibilidad de ocurrencia de un } \\
\text { evento. } \\
\text { - Conjeturo acerca del resultado de un experimento } \\
\text { aleatorio usando proporcionalidad y nociones básicas de } \\
\text { probabilidad. } \\
\text { - Predigo y justifico razonamientos y conclusiones usando } \\
\text { información estadística. }\end{array}$ & $\begin{array}{l}\text { - Resuelvo y formulo } \\
\text { problemas a partir de } \\
\text { un conjunto de datos } \\
\text { presentados en tablas, } \\
\text { diagramas de barras, } \\
\text { diagramas circulares. }\end{array}$ \\
\hline $8^{\circ}-9^{\circ}$ & $\begin{array}{l}\text { - Reconozco cómo diferentes formas de presentación de } \\
\text { información pueden originar distintas interpretaciones. } \\
\text { - Interpreto analítica y críticamente información estadística } \\
\text { proveniente de diversas fuentes (prensa, revistas, televisión, } \\
\text { experimentos, consultas, entrevistas). } \\
\text { - Interpreto y utilizo conceptos de media, mediana y moda } \\
\text { y explicito sus diferencias en distribuciones de distinta } \\
\text { dispersión y asimetría. } \\
\text { - Selecciono y uso algunos métodos estadísticos adecuados } \\
\text { al tipo de problema, de información y al nivel de la escala } \\
\text { en la que esta se representa (nominal, ordinal, de intervalo } \\
\text { o de razón). } \\
\text { - Comparo resultados de experimentos aleatorios con } \\
\text { los resultados previstos por un modelo matemático } \\
\text { probabilístico. } \\
\text { - Reconozco tendencias que se presentan en conjuntos de } \\
\text { variables relacionadas. } \\
\text { - Calculo probabilidad de eventos simples usando } \\
\text { métodos diversos (listados, diagramas de árbol, técnicas } \\
\text { de conteo). } \\
\text { - Uso conceptos básicos de probabilidad (espacio } \\
\text { muestral, evento, independencia, etc.) }\end{array}$ & $\begin{array}{l}\text { Resuelvo y } \\
\text { formulo problemas } \\
\text { s e } 1 \text { e c c i o n a n d o } \\
\text { información relevante } \\
\text { en conjuntos de } \\
\text { datos provenientes } \\
\text { de fuentes (prensa, } \\
\text { revistas, televisión, } \\
\text { ex p e r i m e n t o s, } \\
\text { consultas, entrevistas) }\end{array}$ \\
\hline
\end{tabular}

Fuente: esta investigación; MEN, 2006, 84-88. 


\section{Conclusiones}

Los Indicadores de logros y Lineamientos Curriculares en Colombia para la Educación Básica Secundaria, en relación con el Pensamiento Aleatorio y Resolución de problemas, evidencian, para los diferentes grados de $6^{\circ}$ a $9^{\circ}$, una introducción de elementos y pautas hacia la investigación, desde su formación temprana, aspecto que fundamenta semillas que potencialmente podrán seguir afianzando procesos de formación subsiguientes, como los generados en las Instituciones de Educación Superior, lo que muestra, a su vez, algunas congruencias con la construcción curricular en las instituciones educativas del país.

Se destaca que las principales modificaciones generadas en el currículo en Colombia describen medidas de mejoramiento que propenden hacia avances de comprensión y finalidad de los objetivos de la educación nacional. En ese recorrido histórico de las normativas curriculares en Colombia, se destaca, desde varias décadas atrás, un proceso continuo de cambios que busca normalizar y estandarizar cada vez más los currículos, con objetivos, planes y programas, incididos por referentes internacionales. Así mismo, en la revisión documental, se observa que, si bien hay una serie de normativas, no se evidencian a la vez, por parte del MEN, estudios formales que analicen el efecto o incidencia de los cambios significativos propuestos ni estudios que contrasten con las vivencias y vicisitudes a nivel de las instituciones.

Esta investigación ilustra una estrategia metodológica de organización de información, que permitió identificar, elaborar y presentar, mediante cuadros comprensivos, la descripción histórica de cambios curriculares en temas específicos como el Pensamiento Aleatorio y la Resolución de Problemas Matemáticos para Educación Básica Secundaria. Esta estrategia de organización de información, a su vez, podrá servir de referencia a otros estudios académicos y de investigación sobre otros tópicos de interés particular.

\section{Referencias}

Ayala-García, Jhorland. Evaluación externa y calidad de la educación en Colombia. Cartagena, Colombia: Banco de la República.

Baena Paz, Guillermina. Metodología de la investigación. México: Grupo Editorial Patria, 2017. 
Baena López, Carlos Alberto. "Educación formal vs. Educación Vital”, Diario del Huila, Neiva, 11, septiembre, 2011, 9a . repositorio.unal.edu.cohttps:// repositorio.unal.edu.co/handle/unal/10696

Batanero, Carmen. “¿Hacia dónde va la educación estadística?”. Blaix. No. 15 (2000): 1-14. https://www.ugr.es/ batanero/pages/ARTICULOS/BLAIX.pdf

Batanero, Carmen. Didáctica de la Estadística. Vol. 1. Granada: Universidad de Granada, 2001.

Batanero, Carmen, Juan D. Godino y D. R. Green. "Errores y dificultades en la comprensión de los conceptos estadísticos elementales". International Journal of Mathematics Education in Science and Technology, vol. 25. No. 4 (2014): 1-18. https://www.academia.edu/36641177/Errores_y_dificultades_ en_la_comprensi\%C3\%B3n_ de_los_conceptos_estad\%C3\%ADsticos_ elementales España.

Begué, Nuria et al. "Razonamiento de estudiantes de bachillerato ante una situación binomial". Tangram, Vol. 3. No. 2 (2020): 27-50. doi: 10.30612/ tangram.v3i2.10888.

Beltrán, Diana y Gómez, Nubia. "Propuesta metodológica para analizar y comparar los resultados en pruebas de Estado en las áreas de matemáticas y lenguaje". En: Revolución en la formación y la capacitación para el Siglo XXI, editado por Edgar Serna M. Medellín: Instituto Antioqueño de Investigación, 2020. 598-607.

Benavides, Maryorie et al. "La resolución de problemas en el currículum chileno". Acta Latinoamericana de Matemática Educativa. No. 17 (2004): 807-812. http://funes.uniandes.edu.co/6388/1/BenavidesLaresolucion Alme2004.pdf

Castellanos Sánchez, María Teresa. "Tablas y gráficos estadísticos en la Prueba Saber - Colombia". Trabajo de Máster, Universidad de Granada, 2014. http:// funes.uniandes.edu.co/4750/1/tfm_Castellanos_M_T_final.pdf

Congreso de Colombia. Ley 24 de 1988 (febrero 11). https://www.funcionpublica. gov.co/eva/gestornormativo/norma_pdf.php?i=259

Congreso de la República de Colombia. Ley 115 de febrero 8 de 1994. https:// www.mineducacion.gov.co/1621/ articles-85906_archivo_pdf.pdf 
Currículo. Oxford Léxico. 2020. https://www.lexico.com/es/definicion/curriculo

Departamento de Educación de la Universidad Central Chile. "Investigación Acción: Resolución de problemas matemáticos por parte de estudiantes de Enseñanza Básica de escuelas municipalizadas utilizando diversas estrategias de aprendizaje.” Universidad Central-Unasur. No. 1 (2013): 1-134.

Gómez, Nubia y Rodríguez, Julieth. "Análisis de la producción científica matemática en Colombia. Base Web of Knowledge (2001-2012)". Bistua, vol. 12. No. 2 (2014): 70-84. http://revistas.unipamplona.edu.co/ojs_viceinves/ index.php/BISTUA/article/view/1660/670

Gómez Velasco, Nubia et al. "Análisis de la producción científica colombiana en Química. Base de datos wok (2001-2012)". Revista Logos Ciencia \& Tecnología, vol. 6. No. 1 (2014): 108-115. https://revistalogos.policia.edu. co: 8443/index.php/rlct/article/view/125

Gómez, Nubia y Jiménez, Ana. "La Estadística como apoyo en los proyectos de investigación Universidad-comunidad. Reflexiones de una experiencia con Semilleros de Investigación". Revista Logos Ciencia \& Tecnología, vol. 7. No. 1 (2015): 27-33. https://www.redalyc.org/pdf/5177/517751487003.pdf

Gómez Velasco Nubia et al. Políticas y medición en ciencia y tecnología en la universidad colombiana. 1992-2014. Tunja, Universidad Pedagógica y Tecnológica de Colombia, 2018.

Gómez, Nubia et al. "Elementos de validación y fiabilidad del test Hctaes para estudiar el pensamiento crítico en estudiantes de Psicología". Ponencia. Encuentros Virtual Educa, Perú, 2019. https:/encuentros.virtualeduca.red/ storage/ponencias/ peru2019/ATAUpFazRKGjrOUeYLon3jHwNy7fCpuSl12ny4Ko.pdf

Gómez Velasco, Nubia et al. "Comparación de la eficiencia científica entre Colombia y México a través de indicadores relativos de producción y calidad científica". Revista Española de Documentación Científica, vol. 43. No. 2 (2020): 1-9. https://doi.org/10.3989/redc.2020.2.1644

Gómez, Nubia et al. "Historia y Memoria, casi 10 años consolidando comunidad historiográfica. Una mirada desde la Bibliometría". Historia y Memoria. No. 20 (2020): 209-247. https://revistas.uptc.edu.co/index.php/historia memoria/article/view/9558 
Guerrero Martínez, Katherine y López Vera, Diana. Informe Nacional de Resultados: Colombia en PISA 2015. Bogotá: MEN-Icfes, 2017.

Hernández-Sampieri, Roberto et al. Metodología de la Investigación. México: McGraw-Hill Interamericana Editores, 2017.

Instituto Colombiano para la Evaluación de la Educación. Cuadernillo de Prueba. Ejemplo de Preguntas Saber 9²014. Matemáticas. Bogotá: Icfes, 2016.

Instituto Colombiano para la Evaluación de la Educación. Cuadernillo de Prueba. Ejemplo de Preguntas Saber 9o 2015 Matemáticas. Bogotá: Icfes, 2016.

Instituto Colombiano para la Evaluación de la Educación. Bases de Datos del Icfes. Tipos de pruebas. Bogotá: Icfes, 2017.

Instituto Colombiano para la Evaluación de la Educación. Guía de Orientación Saber $9^{\circ}$. Bogotá: Icfes, 2017.

Instituto Colombiano para la Evaluación de la Educación, Icfes. Guía de Orientación Avancemos 4 $4^{\circ}, 6^{\circ}, 8^{\circ}$ - 2019. Bogotá: Icfes, 2019.

Instituto Colombiano para la Evaluación de la Educación, Icfes. Informe nacional de resultados para Colombia - PISA 2018. Vol. 1. Bogotá: Ministerio de Educación Nacional, 2020.

Instituto Colombiano para la Evaluación de la Educación, Icfes. Guía de Orientación Evaluar para Avanzar $3^{\circ}$ a $11^{\circ}$. Bogotá, 2020. https://www. youtube. $\mathrm{com} /$ watch? $\mathrm{v}=4 \mathrm{~b} 4 \mathrm{xdB} 5 \mathrm{n} 7 \mathrm{zM}$

Jiménez, Ana et al. "Estudio de la colaboración en publicaciones científicas. Facultad de ciencias-UPTC, 2002-2014". Saber, Ciencia y Libertad, vol. 13. No. 1 (2018): 304-318. https://revistas.unilibre.edu.co/index.php/saber/ article/view/2084

Martínez Boom, Alberto et al. "Reformas de la enseñanza en Colombia: 19601980. Del énfasis didáctico al énfasis curricular". Educación y cultura. No. 15 (1988): 12-21. http://www.albertomartinezboom.com/escritos/articulos/ 1988_Reformas_de_la_ensenanza_en_Colombia_1960-1980.pdf

Ministerio de Educación Nacional. Resolución 2343 de junio 5 de 1996. https:// repository.usta.edu.co/bitstream/ handle/11634/280/RESOLUCION_2343_ DE_JUNIO_5_DE_1996.pdf?sequence=21\&isAllowed=y 
Ministerio de Educación Nacional. Lineamientos Curriculares: Matemáticas. Bogotá: Magisterio, 1998.

Ministerio de Educación Nacional. "Estándares curriculares, un compromiso con la excelencia". Altablero. No. 14 (2002). https://www.mineducacion.gov. co/1621/article-87872.html

Ministerio de Educación Nacional. Estándares para la excelencia en la educación. Bogotá: MEN, 2002.

Ministerio de Educación Nacional. La Revolución Educativa. Estándares básicos de Matemáticas y Lenguaje - Educación Básica y Media. Bogotá: MEN: 2003.

Ministerio de Educación Nacional. Estándares Básicos de Competencias en Matemáticas. Bogotá: MEN, 2006.

Ministerio de Educación Nacional. Derechos Básicos de Aprendizaje - DBA. Matemáticas. Vol. 2. Bogotá: Panamericana, 2016.

Ministerio de Educación Nacional. "Preescolar, Básica y Media - Ministerio de Educación Nacional de Colombia.” Portal Preescolar, Básica y Media. 2020. https://www.mineducacion.gov.co/portal/Preescolar-basica-y-media/

Organización para la Cooperación y el Desarrollo Económicos. 2016. Educación en Colombia. Bogotá: MEN, 2016.

Ortiz-Padilla, Myriam et al. "Conocimiento pedagógico del contenido para la enseñanza matemática infantil: Diseño y validación de una escala para su evaluación". En: Aportes a la calidad educativa desde la investigación, editado por Marvel Gravini-Donado et al. (comps.). Barranquilla: Universidad Simón Bolívar, 2017. 233-254.

Pineda Rodríguez, Yheny Lorena y Loaiza Zuluaga, Yasaldez Eder. "Un análisis del trayecto histórico del currículo en Colombia: Segunda Mitad Del Siglo XX”. Revista de Investigaciones, vo1. 17. No. 29 (2017): 150-167. doi: 10.22383/ri.v17i29.94

Presidencia de la República, Colombia. Decreto 1710 de 1963 (julio 25). https:// www.mineducacion.gov.co/1621/ articles-103714_archivo_pdf.pdf 
Presidencia de la República, Colombia. Decreto 088 de 1976 (enero 22). https:// www.mineducacion.gov.co/1759/articles-102584_archivo_pdf.pdf

Presidencia de la República, Colombia. Decreto 1419 de 1978 (julio 17). https:// www.mineducacion.gov.co/1759/articles-102770_archivo_pdf.pdf

Presidencia de la República, Colombia. Decreto 1002 de 1984 (abril 24). https:// www.mineducacion.gov.co/1759/articles-103663_archivo_pdf.pdf

Presidencia de la República, Colombia. Decreto 2647 de 1984 (octubre 24). https://www.mineducacion.gov.co/1621/articles-103689_archivo_pdf.pdf

Presidencia de la República, Colombia. Decreto 1860 de 1994 (agosto 3). https://www.mineducacion.gov.co/1621/ articles-172061_archivo_pdf_ decreto1860_94.pdf

Presidencia de la República, Colombia. Decreto 1290 de 2009 (abril 16). https://www. mineducacion.gov.co/1621/articles-187765_archivo_pdf_decreto_1290.pdf

Presidencia de la República, Colombia. Decreto 5014 de 2009 (diciembre 28). https:// www.funcionpublica.gov.co/eva/gestornormativo/norma_pdf.php?i=67183

¿Qué es la investigación documental? QuestionPro. 2020. https://www. questionpro.com/blog/es/investigacion-documental/

Schleicher, Andrea. PISA 2018: Insigths and Interpretations. París: OECD, 2019.

Vivero, L., y B. I. Sánchez. 2018. La Investigación Documental: características y algunas herramientas. Unidades de Apoyo para el Aprendizaje. CUAED/ Facultad de Arquitectura-UNAM. 2018. http://132.248.48.64/repositorio/ moodle/pluginfile.php/1516/mod_resource/content $/ 3 /$ contenido/index.html

\section{Citar este artículo}

Gómez Velasco, Nubia Yaneth; Beltrán Castañeda, Diana Carolina. "Pensamiento Aleatorio y Resolución de problemas en la Educación Básica Secundaria Normativa Curricular. Una Mirada Histórica". Revista Historia de la Educación Colombiana. Vol. 25 No 25 (2020): 197-219. DOI: https://doi.org/10.22267/ rhec.202525.85 